\title{
Human-Carnivore Conflicts in Private Conservancy Lands of Elerai and Oltiyiani in Amboseli Area, Kenya
}

\author{
Moses Makonjio Okello1*, John Warui Kiringe1, Fiesta Warinwa ${ }^{2}$ \\ ${ }^{1}$ The School for Field Studies, Centre for Wildlife Management Studies, Nairobi, Kenya \\ ${ }^{2}$ African Wildlife Foundation (AWF), Nairobi, Kenya \\ Email: ${ }^{*}$ mokello@fieldstudies.org, ${ }^{*}$ mokello33@gmail.com
}

Received 30 March 2014; revised 3 May 2014; accepted 27 May 2014

Copyright (C) 2014 by authors and Scientific Research Publishing Inc.

This work is licensed under the Creative Commons Attribution International License (CC BY).

http://creativecommons.org/licenses/by/4.0/

c) (i) Open Access

\section{Abstract}

Human-carnivore conflicts represent the most common negative form of interactions between humans and wildlife. Most carnivores involved in such conflicts are: lion, hyena, leopard and cheetah. Three strategies are normally used in Kenya to mitigate such conflicts; consolation for lost livestock and human life to increase tolerance to them, use of predator proof homesteads especially among pastoralists, use flicking lights at night to discourage approach of carnivores near homesteads, and awareness creation among communities on the ecological role of carnivores. This study examined human-carnivore interactions in privately owned conservancies near Amboseli National Park, Kenya. The conservancies were found to have almost similar human and livestock demography. However, homesteads in Elerai had more fence broken parts and relatively higher levels of livestock predation by lion and hyena. The higher the number of each livestock type was, the higher the specific predation to that livestock type was, implying density dependent effects of predation by carnivores on livestock. It seemed that the fence structure and level of maintenance (including carnivore strategies on specializing on specific livestock size and age consistent with optimizing their foraging strategies) influenced predation incidences. However, the presence of adult males and Maasai warriors (morans) in bomas did not seem to be related with the number of livestock killed by carnivores, implying that they didn't add vigilance as an additional strategy to prevent livestock depredation. It is recommended that attention be paid on maintenance of homestead and livestock fences as well as vigilance to deter predation. We further recommend strategies to prevent livestock predation such as, installation of chain link predator proof fences or carnivore lighting deterrents at night because woody plants fences are ineffective and deteriorate easily with time, and also lead to depletion of plant resources critical to households.

\footnotetext{
Corresponding author.
} 


\section{Keywords}

\section{Amboseli, Human-Carnivore Conflicts, Livestock Depredation}

\section{Introduction}

Human-wildlife conflicts, including human-carnivore conflicts, are a leading cause of decline of wildlife populations and contraction of their space throughout Africa (KWS 1994 [1]; Woodroffe 2000 [2]; Patterson et al. 2004 [3]; Maclennan et al. 2009 [4]). It presents one of the most persistent and very emotive aspects of conservation that needs to be managed if humans will co-exist with wildlife especially in poor rural third world countries (Norton-Griffiths and Southey 1995 [5]; Newmark 1994 [6]; Frank 2004 [7]; Kimanai and Pickard 1998 [8]; Okello and Kiringe 2004 [9]; Hazzah et al. 2009 [10]). Of the most animals involved in such conflicts are carnivores, particularly lion Panthera leo, spotted hyena Crocuta crocuta, leopard Panthera pardus, and cheetah Acinonyx jubatus, large mega herbivores especially the African elephant Loxodonta africana, Cape buffalo Syncerus caffer, hippopotamus Hippopotamus amphibius and primates. The manifestation of the conflicts is manifested in different forms such as; crop raiding, livestock predation, spread of wildlife diseases to livestock, destruction of property, human injury and death (KWS 1994 [1]). The main causes for these are probably increasing human population that cause encroachment on wildlife habitats, and competition of critical resources and space between wildlife and humans.

Protected areas provide some of the most important areas available to wildlife legally in the future in Kenya (Gowdy 1997 [11]; Wishitemi and Okello 2003 [12]), but the network of protected areas around the world are faced by all kinds of threat including human encroachment and insularization (Morell 1993 [13]; NewMark 1993 [14], 1996 [15]), mismanagement and insufficient funding (Stuart-Hill and Grossman 1993 [16]). It is therefore important to sustain efforts aimed at expanding space for wildlife outside protected areas, but in safe lands earmarked specifically for conservation such as private and communal wildlife sanctuaries (Graham 1989 [17]; Hackel 1998 [18]; Ferraro and Kiss 2000 [19]; Okello et al. 2003 [20]). However, this initiative needs to be fast-tracked but in a systematic manner since land uses in critical wildlife dispersal areas of major national parks in Kenya and East Africa are rapidly changing making them unsuitable for use by wildlife (Campbell et al. 2000 [21]). This situation is exacerbated by a fast growing sedentary human population which increases encounters with carnivores eventually leading to conflicts, death and ultimately decline in the population of large predators (Mizutani 1993 [22]; Burtler 2000 [23]; Kerbis et al. 2002 [24]; Patterson et al. 2004 [3]).

The consequences for human-carnivore conflicts are evident to both people and wildlife. For the people, it entrenches a negative attitude towards wildlife, lack of support for wildlife conservation and tolerance for sharing space and resources with wildlife (Hazzah et al. 2013 [25]). This is especially the case where predation costs are enormous and persistent, and also uncompensated by the state or conservation agencies. The problem is persistent where there is no interest or capacity by government agencies to control, prevent or fully compensate damages and losses incurred. Ultimately, this sets many local people against conservation initiatives instead of enlisting their support, participation and collaboration. This situation has eventually become one of the biggest impediments to conservation of wildlife and its habitats across many countries of Africa (Galaty 1992 [26]; Norton-Griffiths and Southey 1995 [5]; Gowdy 1997 [11]). It also entrenches rural poverty especially in cases where losses are not controlled, contained or fully compensated for as it transfers the cost and burden of conservation to local communities, when they may not be specifically the biggest beneficiaries of wildlife related benefits (such as tourism revenue and ecosystem services). Similarly, the consequences to the wildlife are serious, and the threats they face from humans may be direct or indirect. Direct consequences involve killing large carnivores through spearing and poisoning to retaliate for the losses incurred, reduced tolerance to wildlife presence including engaging in illegal activities such as bush-meat poaching as a means of reducing wildlife numbers and associated damages. The indirect consequences for negative human interactions include destruction of critical wildlife habitats, encroachment of their space, conversion of their space to alternative land uses, and general insularization. Collectively, indirect and direct consequences have an effect of leading to reduced carnivore populations, local extinctions and their confinement in protected areas (Western 1975 [27]; Rudnai 1979 [28]; Hackel 1998 [18]; Okello et al. 2003 [20]; Patterson et al. 2004 [3]). 
It is therefore imperative that controlling and managing human-wildlife conflicts is an important part of ensuring coexistence between man and beast (KWS 1994 [1]). This co-existence means that there must be consistent and effective means to eliminating or controlling these conflicts. Several strategies have been employed by conservationists and communities to address this with varying degrees of success. In the Amboseli area, the Amboseli for Elephants (ATE) and Big Life Foundation through the elephant research program provides a consolation for livestock killed by elephants outside the parks. In addition, Big Life Foundation provides a consolation to locals whose livestock is killed by large carnivores. These schemes have helped a great deal in restoring local tolerance for wildlife as well as allowing them more range due to community understanding created by these organizations. However, more strategies are needed to complement their effort, such as the new initiatives by Born Free Foundation and African Wildlife Foundation (AWF) who have in the recent past helped construct chain-link predator proof livestock sheds (PPFs) in Maasai homesteads (bomas) within the ecosystem.

Conservation actions like establishment of PPF fences and flicking lights to scare away large carnivores at night in the Amboseli region are addressing the critical question of prevention (before it happens) as well as consolation (after it happens), operating side by side to contain the escalation human-carnivore conflicts. Other general strategies that are also be used include; use of electric fences around agricultural areas, encouraging tourism investments other than agriculture in form of privately owned sanctuaries and establishment of ecolodges whose revenue goes directly to individual land owners. This will augment efforts of the Kenya Wildlife Service (KWS) problem animal management unit (PAMU), community/individual vigilance of farms, as well as use of community game scouts/rangers who help KWS control problem animals and mitigation of human-wildlife conflicts.

This study examined human-carnivore conflicts in the emerging private conservancies in the Amboseli ecosystem. The overall objective was to assess the nature, pattern and extent of the conflicts, and establish the most common problem carnivores. Specifically, the study addressed the following objectives:

1) Assess homestead characteristics, human demography and livestock numbers in homesteads.

2) Evaluate how human demographic characteristics, boma fence integrity and livestock numbers influenced the rate of carnivore predation on livestock in homesteads.

3) Establish the common carnivores that challenged boma fences, and livestock predation incidences in the conservancies.

4) Make recommendations on how to alleviate human-carnivore conflicts in the study sites and other private conservancies in the Amboseli region.

\section{Study Area}

Elerai and Oltiyiani conservancies are part of the Amboseli ecosystem (Figure 1 and Figure 2) which is a renowned wildlife conservation region in Kenya. Wildlife moves seasonally between protected areas (i.e. Tsavo West, Chyulu Hills and Amboseli National Parks) and the adjoining communal group ranches owned by the Maasai (Western 1975 [27]; Wishitemi and Okello 2003 [12]). These wildlife resources have made the ecosystem an important tourism hub, and hence generate a lot of revenue for the country and stakeholders in the tourism industry. The group ranches are part of the ecosystem and collectively provide important wildlife dispersal areas and movement corridors (Western 1975 [27]; Ntiati 2002 [29]; Okello et al. 2003 [20]), including supporting large populations of resident wildlife. In recognition of the value of these communal lands to wildlife survival in the ecosystem, the government though support of USAID established the first private and communally owned wildlife sanctuary (Kimana Community Wildlife Sanctuary) in 1996. Among conservationists, this initiative marked the genesis of establishment of private conservancies in the ecosystem and the country as a whole. It also demonstrated the commitment of the government to mainstream locals in wildlife conservation endeavors whilst involving them in the tourism industry.

Elerai is a privately owned conservancy situated near Amboseli National Park that was started in the 1980's with support from AWF and KWS COBRA Program (Conservation of Bio-diverse Resource Areas) (Figure 2). It borders the Kenya-Tanzania border near and is owned by 8 related families who have set aside their land for wildlife conservation and tourism investment opportunities. Similarly, Oltiyiani is a private conservancy arising from sub-divided land from the former Kimana Group Ranch. It was established in 2000, and is owned by about 85 members (families), and each member has contributed approximately 60 acres of land for the formation of the conservancy. The members opted to merge their individual parcels so as the have multiple gains such as; se- 


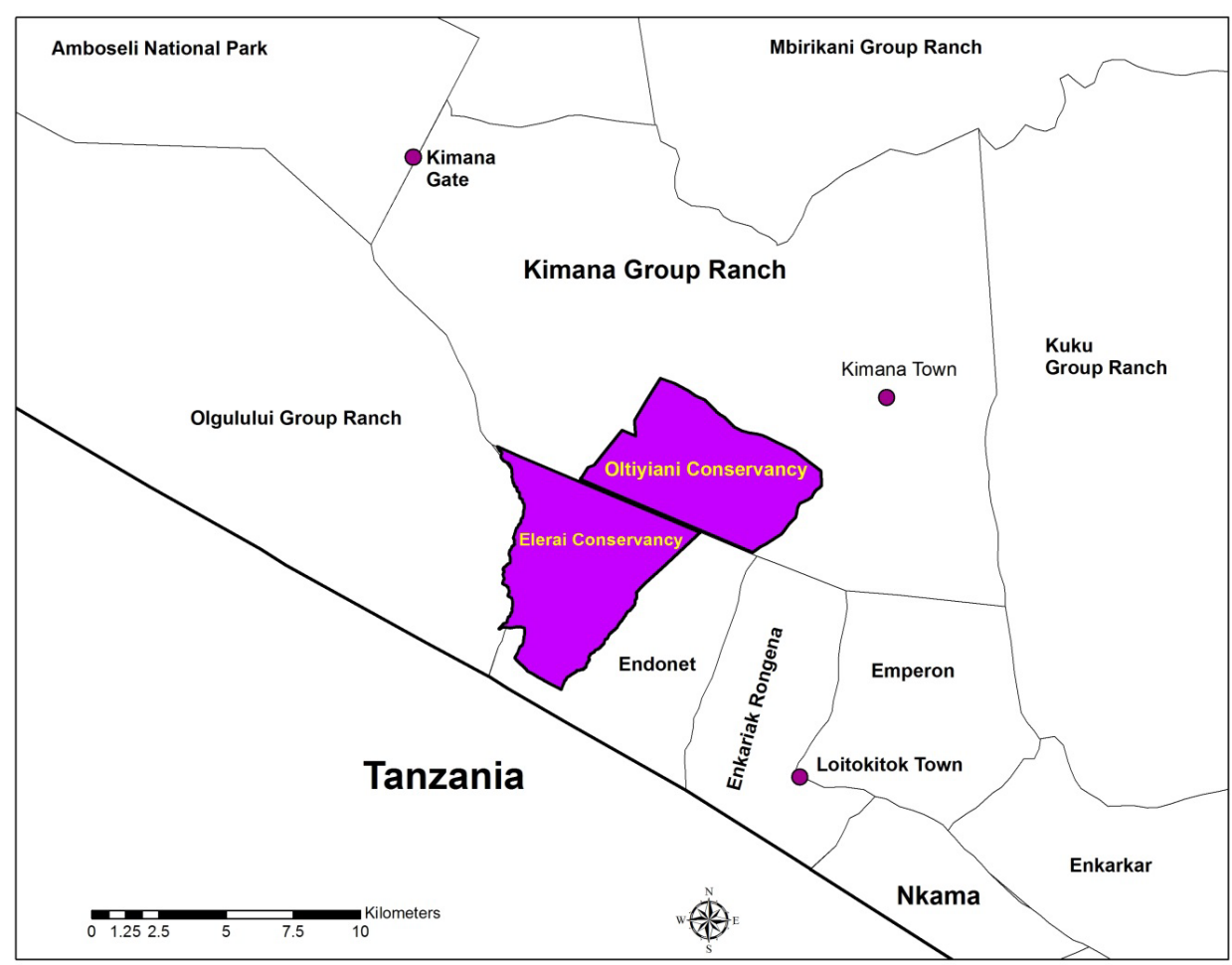

Figure 1. A section of the Amboseli ecosystem showing some of the communal Maasai group ranches in Southern Kenya. Highlighted are Elerai and Oltiyiani Conservancies.

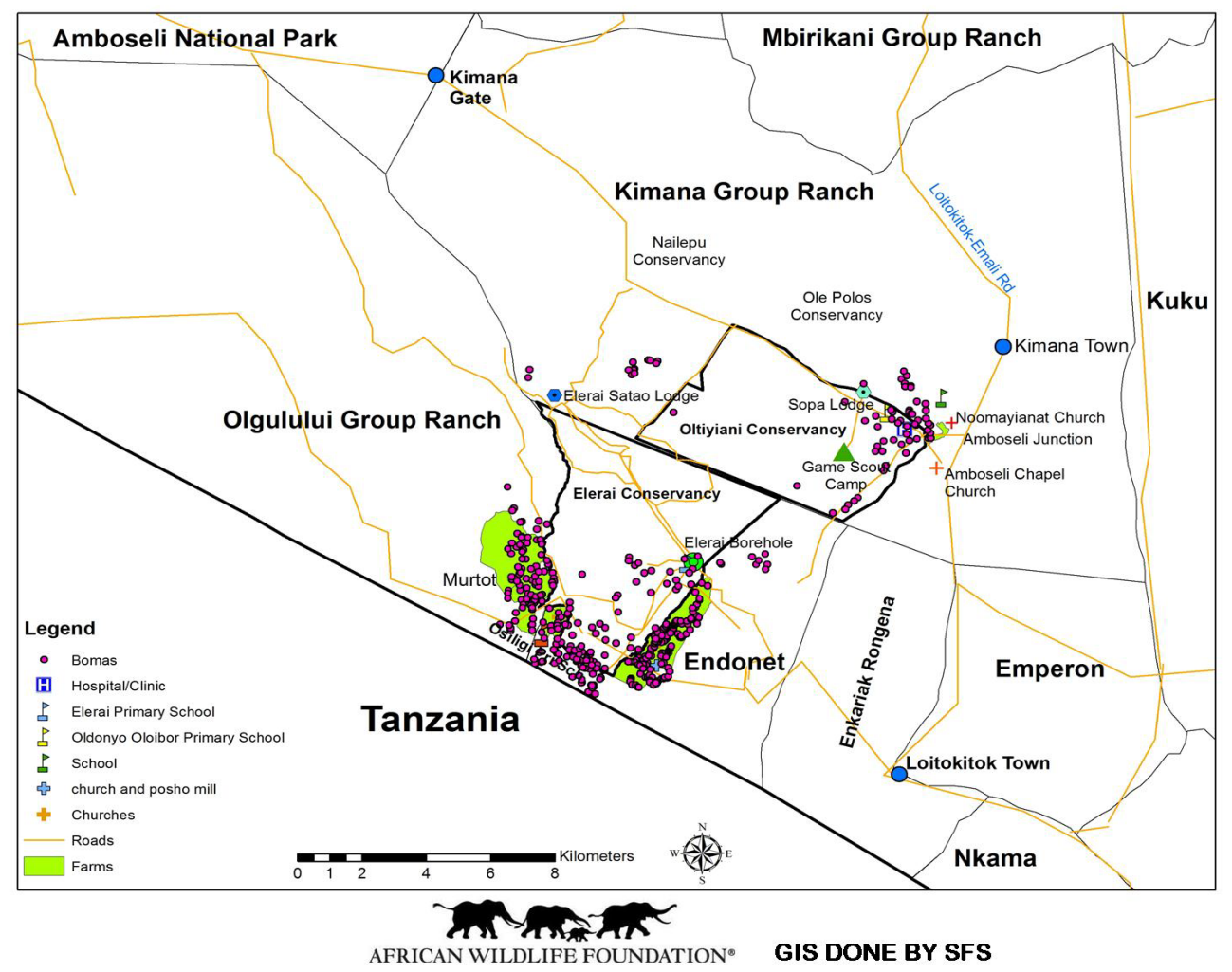

Figure 2. Distribution of Maasai homesteads (bomas) inside and outside the immediate environs of Elerai and Oltiyiani Conservancies as surveyed in the this study. 
curing land for their children, conservation of plant resources to meet their livelihood needs, pastoralism and ecotourism benefits through wildlife conservation.

Most of the Amboseli ecosystem including the two conservancies where this study was done is semi-arid and arid rangeland with a bimodal rainfall pattern (Ntiati 2002 [29]). The long rains occur from around March/April to May while the short rains occur in November and December. The average annual rainfall received within the general locality of the conservancies is between $400 \mathrm{~mm}$ and $500 \mathrm{~mm}$, with $30 \%$ being received during the short rains and 45\% received during the long rains (Irigia 1995 [30]). Soils are classified as volcanic and are generally shallow, highly saline and alkaline (Gachimbi 2002 [31]).

Socio-economic changes in the Amboseli region are increasing demand for group ranch subdivision so that the Maasai can individually feel secure in land ownership. This is further fueled by new government policies which are encouraging dismantling communal ownership of land and nomadic pastoralism into individual ownership (Graham 1989 [17]; Galaty 1992 [26]). These are some of the circumstances which led to the eventual sub-division of Kimana Group Ranch around 2005, and subsequently triggered the creation of private conservancies such as Osupuko, Olepolos, Kilitome, Elerai and Oltiyiani. Unfortunately, this new land tenure policy has increased the opportunity for non-Maasai migrants to lease or even buy the subdivided land hence promoting land uses like agriculture that are not compatible with wildlife conservation. Proliferation of agriculture is therefore an issue of great concern, and is viewed as one of the most serious threats to wildlife conservation in the ecosystem (Pickard 1998 [8]; Ntiati 2002 [29]; Okello and Kiringe 2004 [9]; Okello 2009 [32]).

\section{Methodology}

This study was done between March and June in 2013 in Elerai and Oltiyiani conservancies near Amboseli National Park. We used homesteads as sampling units, and for each we sought the desired information through measurements, observations and interviews with homestead owners. The GPS locations of the homesteads were first established to show their location (Figure 2), after which measurement of boma fence diameter was taken, including making observations on repairs and materials were used to fence the homesteads. Interviews with the homestead owners were done to provide insights on the number of all the people in the homestead, (total people, and number of adult males, women, children and Maasai warriors). Further, information about the number of livestock in general; cattle, shoats (goats and sheep), donkeys and camels were also taken. After this, an interview /discussion with a household male or female leader was done to capture opinions on the effectiveness of the boma fences in deterring livestock predation and human-carnivore conflicts was done in each homestead. Other important information collected included; predators involved in conflicts, livestock predation incidences, compensation sought from killed livestock, and incidences of retaliation killing of large carnivores.

Data analysis was done by calculating averages and variance (Zar 1999 [33]) of common continuous parameters collected. Equality of frequencies was done using Chi-square goodness of fit test, comparisons between some aspects of the conservancies was done using an independent t-test (Zar 1999 [33]), and relationships between fence characteristics and boma demographic variables with livestock killed was done using a Pearson correlation. All tests were deemed significant statistically if the probability of type one error (alpha) was less than 0.05. Tallies and data processing and analysis was done using Excel Microsoft Spreadsheet 2000, and Statistical Package for Social Scientists (SPSS) 2001.

\section{Results}

There were 30 homesteads (bomas) in both Elerai and Oltiyiani, and their distribution was rather clustered (Figure 2). There seemed to be more bomas in the immediate environs of the conservancies than inside. The number of bomas also appeared to more in the northern part of Elerai towards the Kenya-Tanzania border where agriculture was more prevalent but fewer and localized in Oltiyiani (Figure 2). Within Elerai, the highest number of bomas was in Oltinga, followed by Naankamuriak, and lastly Nooloitikoshi, while in Oltiyiani, the highest number of bomas was in Noomayianat near Sopa Lodge, followed by Noonkeshi, and lastly Paranai (Table 1). For Elerai conservancy, the number of families per homestead was $2.36 \pm 0.58$. The average temporary house units per homestead was $4.72 \pm 1.28$, semi-permanent units were $0.90 \pm 0.08$, and permanent units $0.07 \pm$ 0.05 (Table 1). In Oltiyiani, the average number of families per homestead was $1.74 \pm 0.70$ with an average temporary house units per homestead of $2.15 \pm 0.51$, while that of semi-permanent units was $1.25 \pm 0.41$, and permanent units were $0.13 \pm 0.04$ (Table 1 ). 
Table 1. Characteristics of Maasai bomas in Elerai and Oltiyiani conservancies near Amboseli National Park.

\begin{tabular}{|c|c|c|c|c|c|c|}
\hline Conservancy & Location & Total bomas & $\begin{array}{l}\text { Average number of } \\
\text { temporary bomas } \\
\text { (Mean } \pm \text { SE) }\end{array}$ & $\begin{array}{l}\text { Average number of } \\
\text { semi-permanent } \\
\text { bomas (Mean } \pm \text { SE) }\end{array}$ & $\begin{array}{l}\text { Average number of } \\
\text { permanent bomas } \\
\text { (Mean } \pm \text { SE) }\end{array}$ & $\begin{array}{c}\text { Total number of } \\
\text { families } \\
\text { (Mean } \pm \text { SE) }\end{array}$ \\
\hline \multirow{3}{*}{ Elerai } & Naankamuriak & 2 & $\begin{array}{c}4.50 \pm 2.50 \\
\text { Mode: NA } \\
\text { Min/max: } 2,7\end{array}$ & Not present & Not present & $\begin{array}{c}1.00 \pm 0.00 \\
\text { Mode: } 1 \\
\text { Total: } 2 \text { families }\end{array}$ \\
\hline & Nooloitikoshi & 1 & $\begin{array}{l}5.00 \pm 0.00 \\
\text { Mode: NA }\end{array}$ & $\begin{array}{l}2.00 \pm 0.00 \\
\text { Mode: NA }\end{array}$ & Not present & $\begin{array}{c}3.00 \pm 0.00 \\
\text { Mode: NA } \\
\text { Total: } 3 \text { families }\end{array}$ \\
\hline & Oltinga & 27 & $\begin{array}{c}4.65 \pm 1.33 \\
\text { Mode: } 2 \\
\text { Min/max: } 1,37\end{array}$ & $\begin{array}{c}0.70 \pm 0.23 \\
\text { Mode: } 2 \\
\text { Min/max: } 1,2\end{array}$ & $\begin{array}{c}0.22 \pm 0.15 \\
\text { Mode: } 1 \\
\text { Min/max: } 1,4\end{array}$ & $\begin{array}{c}3.07 \pm 1.73 \\
\text { Mode: } 1 \\
\text { Min/max: } 1,48 \\
\text { Total: } 83 \text { families }\end{array}$ \\
\hline \multirow{4}{*}{ Oltiyiani } & Total & 30 & $4.72 \pm 1.28$ & $0.90 \pm 0.08$ & $0.07 \pm 0.05$ & $2.36 \pm 0.58$ \\
\hline & Noonkeshi & 4 & $\begin{array}{c}1.75 \pm 0.48 \\
\text { Mode: } 1 \\
\text { Min/max: } 1,3\end{array}$ & $\begin{array}{c}1.25 \pm 0.75 \\
\text { Mode: NA } \\
\text { Min/max: } 2,3\end{array}$ & Not present & $\begin{array}{c}2.00 \pm 1.00 \\
\text { Mode: } 1 \\
\text { Min/max: } 1,2 \\
\text { Total: } 8 \text { families }\end{array}$ \\
\hline & Noomayianat & 24 & $\begin{array}{c}2.21 \pm 0.54 \\
\text { Mode: } 3 \\
\text { Min/max: } 1,10\end{array}$ & $\begin{array}{c}2.50 \pm 0.47 \\
\text { Mode: } 2 \\
\text { Min/max: } 1,10\end{array}$ & $\begin{array}{c}0.38 \pm 0.13 \\
\text { Mode: } 1 \\
\text { Min/max: } 1,2\end{array}$ & $\begin{array}{c}1.21 \pm 0.10 \\
\text { Mode: } 1 \\
\text { Min/max: } 1,3 \\
\text { Total: } 29 \text { families }\end{array}$ \\
\hline & Paranai & 2 & $\begin{array}{c}2.50 \pm 0.50 \\
\text { Mode: NA } \\
\text { Min/max: } 2,3\end{array}$ & Not present & Not present & $\begin{array}{c}2.00 \pm 1.00 \\
\text { Mode: NA } \\
\text { Min/max: } 1,3 \\
\text { Total: } 4 \text { families }\end{array}$ \\
\hline \multicolumn{2}{|r|}{ Total } & 30 & $2.15 \pm 0.51$ & $1.25 \pm 0.41$ & $0.13 \pm 0.04$ & $1.74 \pm 0.70$ \\
\hline
\end{tabular}

The homesteads' human demographics of interest (number of Maasai warriors (morans), adult males, women, and children) varied between the conservancies. For Elerai, the average number of Maasai warriors per homestead was $4.09 \pm 0.70$, adult males and women was $4.51 \pm 1.73$ and $6.06 \pm 1.33$ respectively while that of children was $24.67 \pm 7.17$ (Table 2). In Oltiyiani, the average number of Maasai warriors per household was $1.85 \pm$ 0.76 , with that of adult males being $2.54 \pm 0.91,3.00 \pm 1.99$ for women and an average of $7.29 \pm 1.99$ for children (Table 2). Overall, there were more people in Nooloitikoshi followed by Naaankamuriank and lastly Oltinga within Elrai conservancy, while in Oltiyiani, there were more people on Noomanayiat followed by Paranai and lastly Noonkeshi (Table 2). In terms of livestock owned, there was an average total livestock of $420.11 \pm 177.87$ and $188.94 \pm 115.07$ per homestead in Elerai and Oltiyiani respectively (Table 3). Further, the average number of cattle per homestead in Elerai was $100.30 \pm 20.54$, for shoats it was $334.81 \pm 163.84$, and donkeys $5.78 \pm$ 2.01. For homesteads in Oltiyiani, the average number of cattle per homestead was $58.03 \pm 47.80$, with an average of $130.74 \pm 69.45$ for shoats, and $0.18 \pm 0.14$ for donkeys (Table 3). Within Elerai, the highest number of livestock was found in Naankamuriak followed by Nooloitikoshi and lastly Oltinga. But in Oltiyiani, the largest number of livestock was found in Paranai followed by Noonkeshi and lastly Noomanayiat.

Several characteristics of the homesteads fences in the two conservancies were obtained (Table 4). Elerai conservancy had an average $5.21 \pm 1.53$ fenced compartments in the homesteads while Oltiyiani had 3.63 \pm 1.19 . The average number of fence broken points in Elerai was $7.42 \pm 0.69$, and fence repairs due to damage were done on average $8.58 \pm 1.47$ times per year. In Oltiyiani, the average number of fence broken points was $3.89 \pm$ 0.95 , while repairs were done $5.25 \pm 1.83$ times per year (Table 4). In Elerai, the largest number of broken fence parts in homesteads was in Oltinga followed by Nooloitikoshi and Naankamuriak in that order. Further, the largest number of fence repairs was done at Nooloitikoshi followed by Naankamuriak, and lastly Oltinga. For Oltiyiani, the highest number of broken fence parts was in Paranai followed by Noomayianat and lastly Noonkeshi. For the number of repairs done, Noomanayiat had the highest followed by Noonkeshi and lastly Paranai

In both Elerai and Oltiyani, more shoats were killed followed by cattle and donkeys every month (Table 5). 
Table 2. Human demographics of Maasai bomas in Elerai and Oltiyiani conservancies near Amboseli National Park.

\begin{tabular}{|c|c|c|c|c|c|}
\hline Conservancy & Location & $\begin{array}{l}\text { Average number of } \\
\text { morans per boma } \\
\text { (Mean } \pm \text { SE) }\end{array}$ & $\begin{array}{l}\text { Average number of } \\
\text { adult men per boma } \\
\text { (Mean } \pm \text { SE) }\end{array}$ & $\begin{array}{l}\text { Average number of adult } \\
\text { women per boma } \\
\text { (Mean } \pm \text { SE) }\end{array}$ & $\begin{array}{l}\text { Average number of } \\
\text { children per boma } \\
\text { (Mean } \pm \text { SE) }\end{array}$ \\
\hline \multirow{3}{*}{ Elerai } & Naankamuriak & $\begin{array}{c}2.50 \pm 1.50 \\
\text { Mode: NA } \\
\text { Min/max: } 1,4\end{array}$ & $\begin{array}{c}5.50 \pm 4.50 \\
\text { Mode: NA } \\
\text { Min/max: } 1,10\end{array}$ & $\begin{array}{c}5.50 \pm 3.50 \\
\text { Mode: NA } \\
\text { Min/max: } 2,9\end{array}$ & $\begin{array}{c}21.00 \pm 19.00 \\
\text { Mode: NA } \\
\text { Min/max: } 2,40\end{array}$ \\
\hline & Nooloitikoshi & $\begin{array}{l}7.00 \pm 0.00 \\
\text { Mode: NA }\end{array}$ & $\begin{array}{l}5.00 \pm 0.00 \\
\text { Mode: NA }\end{array}$ & $\begin{array}{l}9.00 \pm 0.00 \\
\text { Mode: NA }\end{array}$ & $\begin{array}{c}40.00 \pm 0.00 \\
\text { Mode: NA }\end{array}$ \\
\hline & Oltinga & $\begin{array}{c}2.78 \pm 0.59 \\
\text { Mode: } 2 \\
\text { Min/max: } 1,16\end{array}$ & $\begin{array}{c}3.04 \pm 0.70 \\
\text { Mode: } 1 \\
\text { Min/max: } 1,20\end{array}$ & $\begin{array}{c}3.67 \pm 0.50 \\
\text { Mode: } 3 \\
\text { Min/max: } 1,11\end{array}$ & $\begin{array}{c}13.03 \pm 2.51 \\
\text { Mode: } 12 \\
\text { Min/max: } 2,70\end{array}$ \\
\hline \multirow{4}{*}{ Oltiyiani } & otal & $4.09 \pm 0.70$ & $4.51 \pm 1.73$ & $6.06 \pm 1.33$ & $24.67 \pm 7.17$ \\
\hline & Noonkeshi & $\begin{array}{l}1.50 \pm 0.87 \\
\text { Mode: } 3 \\
\text { Min/max: } 3,3\end{array}$ & $\begin{array}{c}2.75 \pm 0.85 \\
\text { Mode: NA } \\
\text { Min/ max: } 1,5\end{array}$ & $\begin{array}{c}2.75 \pm 1.18 \\
\text { Mode: } 1 \\
\text { Min/max: } 1,6\end{array}$ & $\begin{array}{c}6.75 \pm 2.25 \\
\text { Mode: } 3 \\
\text { Min/max: } 3,12\end{array}$ \\
\hline & Noomayianat & $\begin{array}{c}2.04 \pm 0.42 \\
\text { Moe: } 1 \\
\text { Min/max: } 1,9\end{array}$ & $\begin{array}{c}2.38 \pm 0.38 \\
\text { Mode: } 2 \\
\text { Min/max: } 1,8\end{array}$ & $\begin{array}{c}2.75 \pm 0.34 \\
\text { Mode: } 3 \\
\text { Min/max: } 1,7\end{array}$ & $\begin{array}{c}10.13 \pm 1.71 \\
\text { Mode: } 12 \\
\text { Min/max: } 2,40\end{array}$ \\
\hline & Paranai & $\begin{array}{c}2.00 \pm 1.00 \\
\text { Mode: NA } \\
\text { Min/max: } 1,3\end{array}$ & $\begin{array}{c}2.50 \pm 1.50 \\
\text { Mode: NA } \\
\text { Min/max: } 1,4\end{array}$ & $\begin{array}{c}3.50 \pm 0.50 \\
\text { Mode: NA } \\
\text { Min/max: } 3,4\end{array}$ & $\begin{array}{c}5.00 \pm 2.00 \\
\text { Mode: NA } \\
\text { Min/max: } 3,7\end{array}$ \\
\hline \multicolumn{2}{|c|}{ Total } & $1.85 \pm 0.76$ & $2.54 \pm 0.91$ & $3.00 \pm 0.67$ & $7.29 \pm 1.99$ \\
\hline
\end{tabular}

Table 3. Livestock characteristics in Maasai bomas in Elerai and Oltiyiani conservancies near Amboseli National Park.

\begin{tabular}{|c|c|c|c|c|c|}
\hline Conservancy & Location & $\begin{array}{l}\text { Average livestock } \\
\text { per boma } \\
\text { (Mean } \pm \text { SE) }\end{array}$ & $\begin{array}{l}\text { Average number } \\
\text { of cattle per boma } \\
\text { (Mean } \pm \text { SE) }\end{array}$ & $\begin{array}{l}\text { Average number of goats } \\
\text { and sheep(shoats) per boma } \\
\text { (Mean } \pm \text { SE) }\end{array}$ & $\begin{array}{c}\text { Average number of } \\
\text { donkeys per boma } \\
\text { (Mean } \pm \mathrm{SE})\end{array}$ \\
\hline \multirow{3}{*}{ Elerai } & Naankamuriak & $\begin{array}{c}598.00 \pm 512.00 \\
\text { Mode: NA } \\
\text { Min/max: 86, } 1110\end{array}$ & $\begin{array}{c}65.00 \pm 35.00 \\
\text { Mode: NA } \\
\text { Min/max: } 30,100\end{array}$ & $\begin{array}{c}525.00 \pm 475.00 \\
\text { Mode: NA } \\
\text { Min/max: 50, } 1000\end{array}$ & $\begin{array}{c}8.00 \pm 2.00 \\
\text { Mode: NA } \\
\text { Min/max: 6, } 10\end{array}$ \\
\hline & Nooloitikoshi & $\begin{array}{c}556.00 \pm 0.00 \\
\text { Mode: NA }\end{array}$ & $\begin{array}{c}150.00 \pm 0.00 \\
\text { Mode: NA }\end{array}$ & $\begin{array}{c}400.00 \pm 0.00 \\
\text { Mode: NA }\end{array}$ & $\begin{array}{l}6.00 \pm 0.00 \\
\text { Mode: NA }\end{array}$ \\
\hline & Oltinga & $\begin{array}{c}106.33 \pm 21.60 \\
\text { Mode: } 34 \\
\text { Min/max: } 14,350\end{array}$ & $\begin{array}{c}85.89 \pm 26.63 \\
\text { Mode: } 10 \\
\text { Min/max: } 5,100\end{array}$ & $\begin{array}{c}79.42 \pm 16.53 \\
\text { Mode: } 100 \\
\text { Min/max: } 3,300\end{array}$ & $\begin{array}{c}3.34 \pm 4.02 \\
\text { Mode: } 3 \\
\text { Min/max: } 2,20\end{array}$ \\
\hline \multirow{4}{*}{ Oltiyiani } & Total & $420.11 \pm 177.87$ & $100.30 \pm 20.54$ & $334.81 \pm 163.84$ & $5.78 \pm 2.01$ \\
\hline & Noonkeshi & $\begin{array}{c}169.50 \pm 60.87 \\
\text { Mode: NA } \\
\text { Min/max: 40, } 320\end{array}$ & $\begin{array}{c}27.00 \pm 15.67 \\
\text { Mode: NA } \\
\text { Min/max: } 8,70\end{array}$ & $\begin{array}{c}142.50 \pm 49.39 \\
\text { Mode: NA } \\
\text { Min/max: 40, } 250\end{array}$ & Not present \\
\hline & Noomayianat & $\begin{array}{c}108.83 \pm 22.83 \\
\text { Mode: } 50 \\
\text { Min/max: } 27,407\end{array}$ & $\begin{array}{c}20.08 \pm 4.73 \\
\text { Mode: } 20 \\
\text { Min/max: } 2,120\end{array}$ & $\begin{array}{c}88.21 \pm 20.45 \\
\text { Mode: } 30 \\
\text { Min/max: } 15,400\end{array}$ & $\begin{array}{c}0.54 \pm 0.42 \\
\text { Mode: } 2 \\
\text { Min/max: } 1,2\end{array}$ \\
\hline & Paranai & $\begin{array}{l}288.50 \pm 261.50 \\
\text { Mode: NA } \\
\text { Min/max: 27, } 550\end{array}$ & $\begin{array}{c}127.00 \pm 123.00 \\
\text { Mode: NA } \\
\text { Min/max: 4, } 250\end{array}$ & $\begin{array}{c}161.50 \pm 138.50 \\
\text { Mode: NA } \\
\text { Min/max: 23, } 300\end{array}$ & Not present \\
\hline \multicolumn{2}{|c|}{ Total } & $188.94 \pm 115.07$ & $58.03 \pm 47.80$ & $130.74 \pm 69.45$ & $0.18 \pm 0.14$ \\
\hline
\end{tabular}

The number of livestock killed per boma every month in Elerai was $6.34 \pm 1.76$. This was composed of cattle $1.70 \pm 0.27$, shoats $5.92 \pm 1.39$, and donkeys $0.39 \pm 0.23$. On the other hand, the number of livestock killed per boma every month in Oltiyiani was $6.78 \pm 2.88$, which was composed of cattle $1.13 \pm 0.70$, shoats $5.63 \pm 2.30$, and donkeys $0.03 \pm 0.02$. In Elerai, the highest number of livestock killed by carnivores was in Nooloitikoshi followed by Naankamuriak and lastly Oltinga, and for Oltiyiani, the highest number of livestock killed was in 
Table 4. Fence characteristics of Maasai bomas in Elerai and Oltiyiani conservancies near Amboseli National Park.

\begin{tabular}{|c|c|c|c|c|}
\hline Conservancy & Location & $\begin{array}{l}\text { Average number of fence } \\
\text { compartments per boma } \\
\text { (Mean } \pm \text { SE) }\end{array}$ & $\begin{array}{l}\text { Average number of broken } \\
\text { points per boma } \\
\text { (Mean } \pm \text { SE) }\end{array}$ & $\begin{array}{l}\text { Average number of fence } \\
\text { repairs done per year per boma } \\
\text { (Mean } \pm \text { SE) }\end{array}$ \\
\hline \multirow{3}{*}{ Elerai } & Naankamuriak & $\begin{array}{c}6.00 \pm 3.00 \\
\text { Mode: NA } \\
\text { Min/max: } 3,9\end{array}$ & $\begin{array}{c}5.50 \pm 0.50 \\
\text { Mode: NA } \\
\text { Min/max: } 5,6\end{array}$ & $\begin{array}{c}6.50 \pm 3.50 \\
\text { Mode: NA } \\
\text { Min/max: } 3,10\end{array}$ \\
\hline & Nooloitikoshi & $\begin{array}{l}5.00 \pm 0.00 \\
\text { Mode: NA }\end{array}$ & $\begin{array}{l}8.00 \pm 0.00 \\
\text { Mode: NA }\end{array}$ & $\begin{array}{c}13.00 \pm 0.00 \\
\text { Mode: NA }\end{array}$ \\
\hline & Oltinga & $\begin{array}{c}4.63 \pm 1.59 \\
\text { Mode: } 2 \\
\text { Min/max: } 1,40\end{array}$ & $\begin{array}{c}8.77 \pm 1.56 \\
\text { Mode: } 10 \\
\text { Min/max: } 2,30\end{array}$ & $\begin{array}{c}6.23 \pm 0.91 \\
\text { Mode: } 2 \\
\text { Min/max: } 2,20\end{array}$ \\
\hline \multirow{4}{*}{ Oltiyiani } & & $5.21 \pm 1.53$ & $7.42 \pm 0.69$ & $8.58 \pm 1.47$ \\
\hline & Noonkeshi & $\begin{array}{c}3.00 \pm 0.71 \\
\text { Mode: } 4 \\
\text { Min/max: } 1,4\end{array}$ & $\begin{array}{c}3.25 \pm 1.97 \\
\text { Mode: NA } \\
\text { Min/max: } 5,8\end{array}$ & $\begin{array}{c}4.75 \pm 2.43 \\
\text { Mode: } 2 \\
\text { Min/max: } 2,12\end{array}$ \\
\hline & Noomayianat & $\begin{array}{c}3.38 \pm 0.36 \\
\text { Mode: } 3 \\
\text { Min/max: } 2,11\end{array}$ & $\begin{array}{c}4.43 \pm 0.89 \\
\text { Mode: } 2 \\
\text { Min/max: } 1,15\end{array}$ & $\begin{array}{c}8.50 \pm 2.56 \\
\text { Mode: } 3 \\
\text { Min/max: } 1,48\end{array}$ \\
\hline & Paranai & $\begin{array}{c}4.50 \pm 2.50 \\
\text { Mode: NA } \\
\text { Min/max: } 2,7\end{array}$ & $\begin{array}{l}4.00 \pm 0.00 \\
\text { Mode: NA }\end{array}$ & $\begin{array}{c}2.50 \pm 0.50 \\
\text { Mode: NA } \\
\text { Min/max: } 2,3\end{array}$ \\
\hline \multicolumn{2}{|c|}{ Total } & $3.63 \pm 1.19$ & $3.89 \pm 0.95$ & $5.25 \pm 1.83$ \\
\hline
\end{tabular}

Table 5. Livestock killed by large carnivores in Maasai bomas in Elerai and Oltiyiani conservancies near Amboseli National Park.

\begin{tabular}{|c|c|c|c|c|c|}
\hline Conservancy & Location & $\begin{array}{l}\text { Average number of livestock } \\
\text { killed per boma } \\
\text { (Mean } \pm \text { SE) }\end{array}$ & $\begin{array}{l}\text { Average monthly cattle } \\
\text { killed per boma } \\
\text { (Mean } \pm \text { SE) }\end{array}$ & $\begin{array}{l}\text { Average monthly } \\
\text { shoats killed per boma } \\
\text { (Mean } \pm \text { SE) }\end{array}$ & $\begin{array}{l}\text { Average monthly } \\
\text { donkeys killed per } \\
\text { boma (Mean } \pm \text { SE) }\end{array}$ \\
\hline \multirow{3}{*}{ Elerai } & Naankamuriak & $\begin{array}{c}8.50 \pm 4.50 \\
\text { Mode; NA } \\
\text { Min/max: 4, } 13\end{array}$ & $\begin{array}{c}1.50 \pm 0.50 \\
\text { Mode: NA } \\
\text { Min/max: } 1,2\end{array}$ & $\begin{array}{c}6.50 \pm 3.50 \\
\text { Mode: NA } \\
\text { Min/max: } 3,10\end{array}$ & $\begin{array}{l}0.50 \pm 0.50 \\
\text { Mode: NA }\end{array}$ \\
\hline & Nooloitikoshi & $\begin{array}{l}9.00 \pm 0.00 \\
\text { Mode: NA }\end{array}$ & $\begin{array}{l}2.00 \pm 0.00 \\
\text { Mode: NA }\end{array}$ & $\begin{array}{l}7.00 \pm 0.00 \\
\text { Mode: NA }\end{array}$ & Not present \\
\hline & Oltinga & $\begin{array}{c}1.52 \pm 0.79 \\
\text { Mode; } 2 \\
\text { Min/max: 2, } 21\end{array}$ & $\begin{array}{c}1.59 \pm 0.31 \\
\text { Mode; } 1 \\
\text { Min/max: } 1,8\end{array}$ & $\begin{array}{c}4.26 \pm 0.68 \\
\text { Mode: } 3 \\
\text { Min/max: } 1,20\end{array}$ & $\begin{array}{c}0.67 \pm 0.19 \\
\text { Mode: } 1 \\
\text { Min/max: } 1,8\end{array}$ \\
\hline \multicolumn{2}{|c|}{ Total } & $6.34 \pm 1.76$ & $1.70 \pm 0.27$ & $5.92 \pm 1.39$ & $0.39 \pm 0.23$ \\
\hline \multirow{3}{*}{ Oltiyiani } & Noonkeshi & $\begin{array}{c}7.50 \pm 4.63 \\
\text { Mode: NA } \\
\text { Min/max: 4, } 21\end{array}$ & $\begin{array}{c}1.00 \pm 0.41 \\
\text { Mode: } 1 \\
\text { Min/max: } 1,2\end{array}$ & $\begin{array}{c}6.50 \pm 4.56 \\
\text { Mode: } 3 \\
\text { Min/max: 3, } 20\end{array}$ & Not present \\
\hline & Noomayianat & $\begin{array}{c}4.33 \pm 0.50 \\
\text { Mode: } 3 \\
\text { Min/max: } 2,9\end{array}$ & $\begin{array}{c}0.88 \pm 0.20 \\
\text { Mode: } 1 \\
\text { Min/max: } 1,3\end{array}$ & $\begin{array}{c}3.38 \pm 0.33 \\
\text { Mode: } 3 \\
\text { Min/max: } 1,7\end{array}$ & $\begin{array}{c}0.08 \pm 0.06 \\
\text { Mode: } 1\end{array}$ \\
\hline & Paranai & $\begin{array}{c}8.50 \pm 3.50 \\
\text { Mode: NA } \\
\text { Min/max: 5, } 12\end{array}$ & $\begin{array}{l}1.50 \pm 1.50 \\
\text { Mode: NA }\end{array}$ & $\begin{array}{c}7.00 \pm 2.00 \\
\text { Mode: NA } \\
\text { Min/max: 5, } 9\end{array}$ & Not present \\
\hline \multicolumn{2}{|c|}{ Total } & $6.78 \pm 2.88$ & $1.13 \pm 0.70$ & $5.63 \pm 2.30$ & $0.03 \pm 0.02$ \\
\hline
\end{tabular}

Paranai followed by Noonkeshi and lastly Noomanayiat. In regards to fence size dimensions, the average boma diameter in Elerai was $44.19 \pm 4.49$ meters while the average boma area was $1700.21 \pm 386.83 \mathrm{~m}^{2}$ and with an average fence height of $1.29 \pm 0.07$ meters (Table 6). The average boma diameter in Oltiyiani was $42.29 \pm 8.04$ 
Table 6. Average diameter (m) and area of Maasai bomas in Elerai and Oltiyiani conservancies near Amboseli National Park.

\begin{tabular}{|c|c|c|c|c|}
\hline Conservancy & Location & $\begin{array}{l}\text { Average boma diameter (m) } \\
\text { (Mean } \pm \text { SE) }\end{array}$ & $\begin{array}{l}\text { Average boma area (m) } \\
\text { (Mean } \pm \text { SE) }\end{array}$ & $\begin{array}{l}\text { Average fence height (m) } \\
\quad \text { (Mean } \pm \text { SE) }\end{array}$ \\
\hline \multirow{3}{*}{ Elerai } & Naankamuriak & $\begin{array}{c}54.00 \pm 10.50 \\
n=2\end{array}$ & $2376.81 \pm 890.64$ & $1.45 \pm 0.15$ \\
\hline & Nooloitikoshi & $\begin{array}{c}41.50 \pm 0.00 \\
\mathrm{n}=1\end{array}$ & $1352.65 \pm 0.00$ & $1.00 \pm 0.00$ \\
\hline & Oltinga & $\begin{array}{c}37.03 \pm 2.97 \\
\mathrm{n}=27\end{array}$ & $1371.17 \pm 269.86$ & $1.43 \pm 0.05$ \\
\hline \multicolumn{2}{|r|}{ Total } & $\begin{array}{c}44.18 \pm 4.49 \\
n=30\end{array}$ & $1700.21 \pm 386.83$ & $1.29 \pm 0.07$ \\
\hline \multirow{4}{*}{ Oltiyiani } & Noonkeshi & $\begin{array}{c}33.83 \pm 7.04 \\
\mathrm{n}=4\end{array}$ & $3869.31 \pm 1881.83$ & $1.65 \pm 0.06$ \\
\hline & Noomayianat & $\begin{array}{c}39.78 \pm 2.32 \\
n=24\end{array}$ & $3254.63 \pm 432.73$ & $1.45 \pm 0.04$ \\
\hline & Paranai & $\begin{aligned} 53.25 & \pm 14.75 \\
n & =2\end{aligned}$ & $2397.92 \pm 1233.76$ & $1.50 \pm 0.10$ \\
\hline & Total & $\begin{array}{c}42.29 \pm 8.04 \\
n=30\end{array}$ & $3173.95 \pm 618.44$ & $1.53 \pm 0.07$ \\
\hline
\end{tabular}

meters (Table 6). In the same conservancy, the average boma area was $3173.95 \pm 618.44 \mathrm{~m}^{2}$ and the average fence height of $1.53 \pm 0.07$ meters. In Elerai, the fence size (in terms of area) was largest in Naankamuriak followed by Oltinga and lastly at Nooloitikoshi, while in Oltiyiani, the largest fence size was for Noonkeshi followed by Noomanayiat and lastly Paranai.

In both conservancies, three types of large carnivores (lion, spotted hyena and leopard) were frequently mentioned to be involved in conflicts with the locals (Table 7). Their views also showed that within Elerai more livestock was killed by carnivores than Oltiyiani, and this was specifically by lions, while in Oltiyiani hyena kills were the highest. Both conservancies had similar leopard predation on livestock, and for Elerai more livestock kills by carnivore were in Oltinga followed by Naankamuriak and lastly Nooloitikosh. However, lion kills in Elerai were more common in Oltinga followed by Naankamuriak and lastly Nooloitikoshi. For Oltiyiani, the highest livestock killed by carnivores was in Noomanayiat followed by Noonkeshi and lastly Paranai, with hyena kills being highest at Noomanayiat followed by Noonkeshi and least at Parani. Opinions from land owners in the conservancies showed that there were a high number of homesteads $(86.7 \%)$ that repairs were frequently done to keep their boma fences in good working condition (Table 8). Further, a majority of the owners (78.3\%) thought that it was getting harder to acquire the plant materials (thorny bushes) for fence repairs. Many homesteads (96.7\%) noted that the current fence (of thorny woody plants) they are using was not an effective deterrent for predators as they were still losing livestock in predation. A significant majority of members (95.1\%) thought that livestock depredation by large carnivores had increased in the conservancies $\left(\chi^{2}=49.59, \mathrm{df}=1, \mathrm{p}<0.001\right)$, but most (98.4\%) reported never having killed predators in retaliation for livestock losses. Further, a majority (96.7\%) noted that large carnivores always succeeded in their attempts to take livestock from the homesteads at night. A slide majority (61.7\%) of the respondents said that there was no compensation for the loss of livestock to predators in the conservancies, but this number was not significant from those who said that compensation was offered $\left(\chi^{2}=3.27, \mathrm{df}=1, \mathrm{p}=0.07\right)$.

\section{Comparisons of Elerai and Oltiyiani Conservancies}

Comparisons were made between Elerai and Oltiyiani conservancies in terms of homestead characteristics, total livestock present, human demographics and fence characteristics (Table 9). The number of families for both Elerai and Oltiyiani were statistically similar $(\mathrm{t}=1.00 \mathrm{df}=58, \mathrm{p}=0.32)$. Further, the number of adult males, women, morans and children were statistically similar in both conservancies. The number of temporary house units as well as the permanent house units was also statistically similar between Elerai and Oltiyiani conservancies. However, the number of semi-permanent houses in the two conservancies were statistically different $(\mathrm{t}=$ 1.00 , $\mathrm{df}=58, \mathrm{p}=0.32$ ), with Oltiyiani having a higher average of such houses. The average number of people; 
Table 7. Monthly total livestock killed by various carnivore types in Maasai bomas of Elerai and Oltiyiani conservancies near Amboseli National Park.

\begin{tabular}{|c|c|c|c|c|c|c|c|}
\hline Conservancy & Location & $\begin{array}{c}\text { Total } \\
\text { carnivore kills }\end{array}$ & $\begin{array}{c}\text { Lion } \\
\text { livestock kills }\end{array}$ & $\begin{array}{l}\text { Spotted hyena } \\
\text { livestock kills }\end{array}$ & $\begin{array}{c}\text { Leopard } \\
\text { livestock kills }\end{array}$ & $\begin{array}{c}\text { Cheetah } \\
\text { livestock kills }\end{array}$ & $\begin{array}{c}\text { Jackal } \\
\text { livestock kills }\end{array}$ \\
\hline \multirow{3}{*}{ Elerai } & Naankamuriak & 17 & 14 & 3 & - & - & - \\
\hline & Nooloitikoshi & 9 & 6 & 3 & - & - & - \\
\hline & Oltinga & 163 & 99 & 27 & 2 & - & - \\
\hline \multicolumn{2}{|c|}{ Total } & 189 & 119 & 33 & 2 & - & - \\
\hline \multirow{3}{*}{ Oltiyiani } & Noonkeshi & 30 & 5 & 23 & 2 & - & - \\
\hline & Noomayianat & 104 & 39 & 65 & - & - & - \\
\hline & Paranai & 17 & 10 & 7 & - & - & - \\
\hline \multicolumn{2}{|c|}{ Total } & 151 & 54 & 95 & 2 & - & - \\
\hline
\end{tabular}

Table 8. Frequencies of aspects regarding human-carnivore conflicts in Elerai and Oltiyiani conservancies near Amboseli National Park.

\begin{tabular}{|c|c|c|c|c|c|}
\hline Number & Issue being investigated & $\begin{array}{c}\text { Aspect } \\
\text { outcomes }\end{array}$ & Frequencies & Percentages & $\begin{array}{l}\text { Chi-square goodness } \\
\text { of fit test outcome }\end{array}$ \\
\hline \multirow{2}{*}{1} & \multirow{2}{*}{$\begin{array}{l}\text { Opinion on whether repairs have been } \\
\text { done on the broken points on fences }\end{array}$} & Yes & 52 & 86.7 & \multirow{2}{*}{$\begin{array}{c}\chi^{2}=32.27 \\
\text { d.f }=1 \\
p<0.001\end{array}$} \\
\hline & & No & 8 & 13.3 & \\
\hline \multirow{3}{*}{2} & \multirow{3}{*}{$\begin{array}{l}\text { Opinion on how far members } \\
\text { go to gather fencing materials }\end{array}$} & Near & 16 & 26.2 & \multirow{3}{*}{$\begin{array}{c}\chi^{2}=1.61 \\
\text { d.f }=2 \\
p=0.45\end{array}$} \\
\hline & & Far & 21 & 34.4 & \\
\hline & & Very far & 24 & 39.3 & \\
\hline \multirow{2}{*}{3} & \multirow{2}{*}{$\begin{array}{l}\text { Opinion on whether it was getting hard to acquire } \\
\text { plant materials for making and repairing fences }\end{array}$} & Yes & 47 & 78.3 & \multirow{2}{*}{$\begin{array}{l}\chi^{2}=19.27 \\
\text { d.f }=1 \\
p<0.001\end{array}$} \\
\hline & & No & 13 & 21.7 & \\
\hline \multirow{2}{*}{4} & \multirow{2}{*}{$\begin{array}{l}\text { Opinion on whether large carnivores succeeded } \\
\text { in taking livestock from bomas at night }\end{array}$} & Yes & 59 & 96.7 & \multirow{2}{*}{$\begin{array}{l}\chi^{2}=53.26 \\
\text { d.f }=1 \\
p<0.001\end{array}$} \\
\hline & & No & 2 & 3.3 & \\
\hline \multirow[t]{2}{*}{5} & \multirow{2}{*}{$\begin{array}{l}\text { Opinion on the trend of carnivore attacks } \\
\text { on livestock in the entire conservancy }\end{array}$} & Same & 3 & 4.9 & \multirow{2}{*}{$\begin{array}{c}\chi^{2}=49.59 \\
\text { d.f }=1 \\
p<0.001\end{array}$} \\
\hline & & Has increased & 58 & 95.1 & \\
\hline \multirow[t]{2}{*}{6} & \multirow{2}{*}{$\begin{array}{c}\text { Opinion on whether anyone in the bomas } \\
\text { had been forced to defend livestock by } \\
\text { killing predators in retaliation }\end{array}$} & Yes & 1 & 1.6 & \multirow{2}{*}{$\begin{array}{l}\chi^{2}=57.07 \\
\text { d.f }=1 \\
p<0.001\end{array}$} \\
\hline & & No & 60 & 98.4 & \\
\hline \multirow{2}{*}{7} & \multirow{2}{*}{$\begin{array}{l}\text { Opinion on whether compensation was done } \\
\text { for livestock killed by large carnivores }\end{array}$} & Yes & 23 & 38.3 & \multirow{2}{*}{$\begin{array}{c}\chi^{2}=3.27 \\
\text { d.f }=1 \\
p=0.07\end{array}$} \\
\hline & & No & 37 & 61.7 & \\
\hline
\end{tabular}

morans (warriors), adult males, women, and children present in each boma were statistically similar in both conservancies.

The average number of cattle and shoats were also similar for the two conservancies (Table 9). Elerai however, had a significantly higher number of donkeys compared to Oltiyiani. It also had a significant higher number of cattle and donkeys killed per household every month by carnivores compared to Oltiyiani. The average number of shoats killed per household per month was statistically similar between the conservancies. Of the three main predators that were reported to kill livestock, lions had a significantly higher number of kills in Elerai than in Oltiyiani. However, the spotted hyenas and leopard did not show any statistical difference in number of kills between the two conservancies. Aspects of the enclosures (boma) and their fences were also compared (Table 9). Among the parameters considered, the number of fence compartments, the longest fence diameter, 
Table 9. Comparisons of Maasai homesteads in terms of human demographics, livestock predation by carnivores and fence characteristics in Elerai and Oltiyiani conservancies.

\begin{tabular}{|c|c|c|c|}
\hline Variables & $\begin{array}{l}\text { Conservancy means } \\
\quad(\text { Means } \pm \text { SE })\end{array}$ & Statistical outcomes & Conclusions \\
\hline Number of families present per boma & $\begin{array}{c}\text { Elerai }=2.93 \pm 1.56 \\
\text { Oltiyiani }=1.37 \pm 0.16\end{array}$ & $t=1.00 ;$ d.f $=58 ; p=0.32$ & Similar \\
\hline Temporary houses per boma & $\begin{array}{c}\text { Elerai }=4.66 \pm 1.22 \\
\text { Oltiyiani }=2.17 \pm 0.43\end{array}$ & $t=1.95 ;$ d.f $=57 ; p=0.056$ & Similar \\
\hline Semi-permanent house units present per boma & $\begin{array}{c}\text { Elerai }=0.70 \pm 0.21 \\
\text { Oltiyiani }=2.17 \pm 0.40\end{array}$ & $t=-3.22 ;$ d.f $=43.57 ; p=0.002$ & $\begin{array}{l}\text { Significant } \\
\text { difference }\end{array}$ \\
\hline Permanent house units present per boma & $\begin{array}{c}\text { Elerai }=0.20 \pm 0.14 \\
\text { Oltiyiani }=0.30 \pm 0.11\end{array}$ & $t=-0.57 ;$ d.f $=58 ; p=0.57$ & Similar \\
\hline Cattle present per boma & $\begin{array}{c}\text { Elerai }=33.30 \pm 6.88 \\
\text { Oltiyiani }=28.13 \pm 8.75\end{array}$ & $t=0.46 ;$ d.f $=58 ; p=0.64$ & Similar \\
\hline Shoats present per boma & $\begin{array}{c}\text { Elerai }=121.21 \pm 36.55 \\
\text { Oltiyiani }=100.33 \pm 19.07\end{array}$ & $\mathrm{t}=0.51 ; \mathrm{d} . \mathrm{f}=57 ; \mathrm{p}=0.61$ & Similar \\
\hline Donkeys present per boma & $\begin{array}{l}\text { Elerai }=3.76 \pm 0.75 \\
\text { Elerai }=0.43 \pm 0.34\end{array}$ & $t=4.04 ;$ d.f $=38.96 ; p<0.01$ & $\begin{array}{l}\text { Significant } \\
\text { difference }\end{array}$ \\
\hline Warriors (morans) present per boma & $\begin{array}{c}\text { Elerai }=2.90 \pm 0.55 \\
\text { Oltiyiani }=1.97 \pm 0.36\end{array}$ & $t=1.42 ;$ d.f $=58 ; p=0.16$ & Similar \\
\hline Adult men present per boma & $\begin{array}{c}\text { Elerai }=3.27 \pm 0.68 \\
\text { Oltiyiani }=2.43 \pm 0.33\end{array}$ & $\mathrm{t}=1.11 ; \mathrm{d} . \mathrm{f}=58 ; \mathrm{p}=0.27$ & Similar \\
\hline Adult women per boma & $\begin{array}{c}\text { Elerai }=3.97 \pm 0.52 \\
\text { Oltiyiani }=2.80 \pm 0.31\end{array}$ & $t=1.93 ;$ d.f $=58 ; p=0.06$ & Similar \\
\hline Children present per boma & $\begin{array}{c}\text { Elerai }=14.47 \pm 2.61 \\
\text { Oltiyiani }=9.33 \pm 1.42\end{array}$ & $\mathrm{t}=1.73 ; \mathrm{d} . \mathrm{f}=58 ; \mathrm{p}=0.09$ & Similar \\
\hline Fence compartments present per boma & $\begin{array}{c}\text { Elerai }=4.43 \pm 1.29 \\
\text { Oltiyiani }=3.40 \pm 0.33\end{array}$ & $t=0.78 ;$ d.f $=58 ; p=0.44$ & Similar \\
\hline Fence diameter (meters) of per boma & $\begin{array}{c}\text { Elerai }=42.36 \pm 2.93 \\
\text { Oltiyiani }=44.14 \pm 4.37\end{array}$ & $t=-0.35 ;$ d.f $=40 ; p=0.73$ & Similar \\
\hline Average height (meters) of bomas & $\begin{array}{c}\text { Elerai }=1.42 \pm 0.04 \\
\text { Oltiyiani }=1.48 \pm 0.03\end{array}$ & $t=-1.11$ d.f $=57 ; p=0.27$ & Similar \\
\hline Average number of broken points in the fence per boma & $\begin{array}{c}\text { Elerai }=8.43 \pm 1.32 \\
\text { Oltiyiani }=4.25 \pm 0.79\end{array}$ & $\mathrm{t}=2.73 ; \mathrm{d} . \mathrm{f}=44.08 ; \mathrm{p}=0.009$ & $\begin{array}{l}\text { Significant } \\
\text { difference }\end{array}$ \\
\hline Average number of fence repairs done per year per boma & $\begin{array}{c}\text { Elerai }=6.14 \pm 0.84 \\
\text { Oltiyiani }=7.60 \pm 2.09\end{array}$ & $t=-0.63 ;$ d.f $=56 ; p=0.53$ & Similar \\
\hline Average number of cattle killed per month per boma & $\begin{array}{c}\text { Elerai }=1.60 \pm 0.20 \\
\text { Oltiyiani }=0.93 \pm 0.19\end{array}$ & $t=1.98 ;$ d.f $=58 ; p=0.05$ & $\begin{array}{l}\text { Significant } \\
\text { difference }\end{array}$ \\
\hline Average number of shoats killed per month per boma & $\begin{array}{c}\text { Elerai }=4.50 \pm 0.65 \\
\text { Oltiyiani }=4.03 \pm 0.65\end{array}$ & $\mathrm{t}=0.51 ; \mathrm{d} . \mathrm{f}=58 ; \mathrm{p}=0.61$ & Similar \\
\hline Average number of donkeys killed per month per boma & $\begin{array}{c}\text { Elerai }=0.63 \pm 0.18 \\
\text { Oltiyiani }=0.07 \pm 0.05\end{array}$ & $\mathrm{t}=3.11 ; \mathrm{d} . \mathrm{f}=33.00 ; \mathrm{p}=0.004$ & $\begin{array}{l}\text { Significant } \\
\text { difference }\end{array}$ \\
\hline Average monthly livestock kills by a lion per boma & $\begin{array}{c}\text { Elerai }=3.97 \pm 0.41 \\
\text { Oltiyiani }=1.80 \pm 0.28\end{array}$ & $t=4.33 ;$ d.f $=58 ; p=0.001$ & $\begin{array}{l}\text { Significant } \\
\text { difference }\end{array}$ \\
\hline Average monthly livestock kills by spotted hyena per boma & $\begin{array}{c}\text { Elerai }=2.27 \pm 0.40 \\
\text { Oltiyiani }=3.17 \pm 0.61\end{array}$ & $t=-1.24 ;$ d.f $=58 ; p=0.22$ & Similar \\
\hline Average monthly livestock kills by leopard per boma & $\begin{array}{c}\text { Elerai }=0.07 \pm 0.07 \\
\text { Oltiyiani }=\text { None }\end{array}$ & $t=1.00 ;$ d.f $=29 ; p=0.33$ & Similar \\
\hline Average monthly livestock kills by cheetah per boma & $\begin{array}{c}\text { Elerai }=\text { None } \\
\text { Oltiyiani }=0.07 \pm 0.07\end{array}$ & $t=11.00 ; d . f=29 ; p=0.33$ & Similar \\
\hline
\end{tabular}

the average outer fence height, and the number of repairs carried out on the fences annually per household were statistically similar in the conservancies. However, Elerai had statistically higher average number of broken points on the fences per boma compared to Oltiyiani. The correlation between family size and demographics (number of adult males, women, morans and number of children) had large positive correlation (Table 10) with 
Table 10. Correlation of livestock killed with human and livestock demographics, and fence features.

\begin{tabular}{|c|c|c|c|}
\hline & Average monthly cattle killed & Average monthly shoats killed & Average donkeys cattle killed \\
\hline \multicolumn{4}{|l|}{ Human Demographics } \\
\hline Number of families & $\begin{array}{c}r=0.68^{* *} \\
\text { d.f }=59 \\
p<0.001\end{array}$ & $\begin{array}{c}r=-0.38 \\
\text { d.f }=59 \\
p=0.77\end{array}$ & $\begin{array}{l}r=0.29^{*} \\
\text { d.f }=59 \\
p=0.03\end{array}$ \\
\hline Number of morans & $\begin{array}{c}r=0.61^{* *} \\
\text { d.f }=59 \\
p<0.001\end{array}$ & $\begin{array}{l}r=0.05 \\
\text { d.f }=59 \\
p=0.03\end{array}$ & $\begin{array}{l}r=0.32^{*} \\
\text { d.f }=59 \\
p<0.01\end{array}$ \\
\hline Number of adult males & $\begin{array}{c}r=0.62^{* *} \\
\text { d.f }=59 \\
p<0.001\end{array}$ & $\begin{array}{l}r=0.13 \\
d . f=59 \\
p=0.31\end{array}$ & $\begin{array}{l}r=0.24 \\
d . f=59 \\
p=0.07\end{array}$ \\
\hline Number of adult women & $\begin{array}{c}r=0.44 \\
\text { d.f }=59 \\
p<0.001\end{array}$ & $\begin{array}{l}r=0.10 \\
\text { d.f }=59 \\
p<0.45\end{array}$ & $\begin{array}{l}r=0.17 \\
d . f=59 \\
p=0.20\end{array}$ \\
\hline Number of children & $\begin{array}{l}r=0.15 \\
d . f=59 \\
p=0.26\end{array}$ & $\begin{array}{l}r=0.13 \\
d . f=59 \\
p=0.34\end{array}$ & $\begin{array}{c}r=0.45^{* *} \\
d . f=59 \\
p<0.001\end{array}$ \\
\hline \multicolumn{4}{|l|}{ Livestock Demographics } \\
\hline Number of cattle in the boma & $\begin{array}{l}r=0.30^{*} \\
\text { d.f }=59 \\
p=0.02\end{array}$ & $\begin{array}{l}r=0.23 \\
d . f=59 \\
p=0.07\end{array}$ & $\begin{array}{l}r=0.08 \\
d . f=59 \\
p=0.54\end{array}$ \\
\hline Number of shoats in the boma & $\begin{array}{c}r=0.61^{* *} \\
\text { d.f }=58 \\
p=0.15\end{array}$ & $\begin{array}{c}r=0.43^{* *} \\
d . f=58 \\
p=0.001\end{array}$ & $\begin{array}{l}r=0.08 \\
d . f=58 \\
p=0.55\end{array}$ \\
\hline Number of donkeys in the boma & $\begin{array}{c}r=0.57^{* *} \\
\text { d.f }=58 \\
p<0.001\end{array}$ & $\begin{array}{l}r=0.08 \\
d . f=58 \\
p=0.54\end{array}$ & $\begin{array}{c}r=0.47^{* *} \\
d . f=58 \\
p<0.001\end{array}$ \\
\hline \multicolumn{4}{|l|}{ Boma characteristics and parameters } \\
\hline Longest Diameter & $\begin{array}{c}r=0.50^{* *} \\
\text { d.f }=41 \\
p=0.001\end{array}$ & $\begin{array}{l}r=0.28 \\
\text { d.f }=41 \\
p=0.07\end{array}$ & $\begin{array}{l}r=0.12 \\
\text { d. } f=41 \\
p=0.47\end{array}$ \\
\hline Boma Radius & $\begin{array}{l}r=0.07 \\
d . f=17 \\
p=0.77\end{array}$ & $\begin{array}{c}r=0.68^{* *} \\
d . f=17 \\
p=0.002\end{array}$ & $\begin{array}{c}r=-0.37 \\
\text { d.f }=17 \\
p=0.13\end{array}$ \\
\hline Average main outer fence height & $\begin{array}{l}r=0.07 \\
\text { d.f }=58 \\
p=0.59\end{array}$ & $\begin{array}{l}r=0.11 \\
d . f=58 \\
p=0.42\end{array}$ & $\begin{array}{l}r=0.09 \\
d . f=58 \\
p=0.52\end{array}$ \\
\hline Number of broken points & $\begin{array}{c}r=-0.19 \\
d . f=55 \\
p=0.17\end{array}$ & $\begin{array}{l}r=0.03 \\
\text { d.f }=55 \\
p=0.81\end{array}$ & $\begin{array}{c}r=-0.105 \\
\text { d.f }=55 \\
p=0.44\end{array}$ \\
\hline Number of times repaired per year & $\begin{array}{l}r=0.07 \\
d . f=57 \\
p=0.65\end{array}$ & $\begin{array}{l}r=0.05 \\
d . f=57 \\
p=0.73\end{array}$ & $\begin{array}{l}r=0.01 \\
\text { d.f }=57 \\
p=0.95\end{array}$ \\
\hline Number of compartments & $\begin{array}{l}r=0.16 \\
d . f=59 \\
p=0.21\end{array}$ & $\begin{array}{l}r=0.02 \\
d . f=59 \\
p=0.93\end{array}$ & $\begin{array}{l}r=0.15 \\
d . f=59 \\
p=0.24\end{array}$ \\
\hline
\end{tabular}

the number of livestock killed, except the negative (but insignificant) relationship between the number of families and the monthly average number of shoats killed $(r=-0.38$, $\mathrm{df}=59, \mathrm{p}=0.77)$. The number of families, morans, adult males and women had a significant positive relationship with the number of cattle killed by large predators ( $p<0.05$ in all cases). For shoats, the positive significant relationship was with the morans, while for the donkeys, the positive significant relationship was with the number of families, the number of morans and the 
number of children ( $\mathrm{p}<0.05$ in all cases).

In terms of livestock kills, there was a positive and significant ( $p<0.05$ in all cases) correlation between livestock type and its predation number. Hence, an increase in numbers of cattle led to more predation on cattle and vice versa, an increase in the number of shoats led to more predation on shoats and vice versa, and an increase in the number of donkey led to more predation on the donkeys (Table 10). On homestead size, there was generally a positive correlation between homestead size and the number of livestock (cattle, shoats and donkeys) average monthly kills (Table 10). The only significant positive correlation $(\mathrm{p}=0.001)$ was between the diameter of the boma and the number of cattle killed. Fence repair parameters such as number of broken fence parts and repairs per month, though positively correlated with number of various livestock predation; the relationship was not significant ( $p>0.05$ in all cases). Most of the correlations between human demographics and the number of livestock killed were positive (Table 9). These implies that increase in number of families in a household, number of Maasai warriors, number of adult males and women, and the number of children was associated with an increase in the number of livestock killed. The only exception was the negative correlation (implying increase in number of family per household was associated with a decrease in the predation rates) between the number of families and the average number of shoats killed per month, even though this relationship was not significant ( $\mathrm{r}$ $=-0.38, \mathrm{df}=59, \mathrm{p}=0.77$ ). The positive relationship between number of families, number of morans and number of adult males on one hand, and cattle killed were all significant $(\mathrm{p}<0.05)$. Further, the number of cattle, shoats, and donkeys per boma had significant positive correlation with their respective average monthly kills (Table 9), implying that as the number of each of the livestock type increased, the number of kills of that type by large carnivores also increased, and when the number reduced, the carnivore kills for that particular livestock type also declined.

\section{Discussion}

The findings of this study revealed that the conservancies had similar number of families and most housing characteristics. Similarity was also found in number of household demographics (adult males, women, children and morans). Further, the number of livestock and its types (except cattle which were higher in Elerai) were similar. These findings are consistent with what would be expected because most Maasai homes are similar in their arrangement, structure and pattern in terms of sharing a single homestead (boma). Elerai conservancy also had the largest number of broken fences and livestock predation rates, especially from lions. This confirms that the number of broken fences, fence structure, and fence maintenance and fence condition may be the single most important determinant of predation. This predation rates, being density dependent, will increase with the number of livestock in a boma because it provides more successful opportunities for predation by carnivores than bomas that have fewer livestock in well maintained fences. Lions particularly will be most successful in such attacks, but so will hyenas since they normally dig under a fence and access livestock. The abundance and prey preference weight range still gives the hyena a broad and wider choice for livestock that it can attack, making it the most prevalent and leading carnivore problem animal in the conservancies.

It is expected that the security of livestock implies security of wealth and social capital for the Maasai and therefore more families are likely to prefer to stay together in a bigger fenced homestead to escape livestock losses and general insecurity posed to them by large carnivores. Since homesteads usually comprise of more than one family related or not living together, it is not surprising that the number of families are expected to be higher in a bigger fenced homestead. The number of people will go together with more livestock (together with livestock holding pens and layers of fence for additional protection against predation), particularly cattle and shoats which are more economically and socially valuable form of livestock to the Maasai. The number of livestock will increase with presence of more families, but will also increase with perceived and real security and protection from carnivores due to the physical barrier afforded by a better fenced boma and maintained fence in areas not frequented by carnivores.

Information gathered from the respondent's showed that hyena, lion, and the leopard were the most common problem species in regard to livestock attacks. Lions were more common, especially in Elerai which lies close to Amboseli Naitonal Park, while hyena is widespread in the region. It is not surprising therefore that these carnivores will be the common problem carnivore in these conservancies. However, those interviewed also mentioned that in addition to lion, hyena, and leopard, cheetahs and baboons also killed their livestock. Generally, the predation effects of the carnivores were influenced by their numbers, numbers of competing carnivores, the 
state of the fence and the livestock type. An in increase in density of a particular carnivore will lead to more incidences of livestock predation, and depressed effect on competing carnivore numbers due to natural prey competition and intra-competitor sabotage (Estes 1997 [34]). A weak and badly maintained fence will not provide an effective deterrent to predators taking livestock. Further, the vulnerability of livestock to a particular predator depends on the size of the livestock type, and larger carnivores have a tendency of taking larger prey than small-sized carnivores. Therefore, the lion and hyena will easily take cattle and donkey more often than a jackal or a cheetah. However, in terms of optimizing returns from forage, lions will not prefer small prey like shoats which are easily and frequently taken by cheetahs and jackals. But generally, sick and young animals, as well as solitary ones not properly protected will be easy targets for all the carnivores.

It's evident from the interviews with homestead owners that for fences to be effective deterrents to predators, regular and good maintenance are critical, and this partly explains their success in reducing livestock predation. This fact must continue to be emphasized to the Maasai as a critical component of anti-predation strategies aimed at reducing human-carnivore conflicts in the conservancies. But most critical in mitigating this problem is a combination of fence types and materials used (i.e. thorny woody plants and chain link predator proof fences), as well as deterrents such as night flicking lights that will scare away and discourage carnivores closer to bomas.

It was expected from this study that the number of people especially adult males and Maasai warriors' presence in a boma would provide additional protection against predation of livestock by carnivores. Thus, it was envisaged that there would be a significant and negative correlation between the number of people, adult males and Maasai warriors with the number of livestock killed in homesteads. However, from the results, most correlations were positive, and those which were negative (i.e. number of morans and adult males with donkeys killed in bomas) were few. This unexpected finding suggests that the number of people or adult males or Maasai warriors is not in themselves key deterrents against predation of livestock in a Maasai homestead whether it's fenced or not. Perhaps, this may be due to the fact that they do not keep vigilance at night but generally react when livestock attacks have occurred. It also suggests that the number of people in a boma is more associated with high livestock numbers other than providing additional security for livestock. Overall, these findings lead to the conclusion that it is the physical state of the thorny fences and its effectiveness (in structure, supported by good maintenance and repair) that makes them effective deterrents and not additional vigilance by humans at night. Nevertheless, this aspect needs further research.

It is instructive however that the correlations between broken fence and livestock killed was positive (though not significant) implying that increase in fence damage will lead to more predation event by carnivores. This strengthens the view that the effectiveness of a fence is closely linked with its structural integrity, proper and frequent repair, and its hence a critical factor as a mitigation strategy against livestock predation and reduction in human-predator conflicts. The number of broken fence parts, deterioration of thorny plant materials the Maasai's use collectively weaken its barrier integrity and ultimately leads to higher livestock predation rates. In discussions with owners of the homesteads, they indicated however, that their natural thorny plant vegetation was ineffective in reducing and deterring livestock predation from carnivores and majority desired the chain link predator proof fence. Further, it is getting harder to access plant resources for this maintenance and so most carnivores succeed to challenge the fence and take livestock. This use of thorny plants for fencing has huge degradation effects on the vegetation and environment as well (Kiringe and Okello 2005 [35]). In this regard, we recommend that this request be prioritized to match funds availability and be given to homesteads with highest predation incidences (near parks and inside private and community conservancies).

\section{Conclusions}

In conclusion, the frequent carnivore problem animals in the two conservancies were: hyena, lion, jackals, leopard, cheetah and baboons in some cases respectively. This is also consistent with the study by Maclennan et al. (2009 [36]). To prevent their predation effects, the chain link fence in addition to emerging strategies like installing flicking lights around homesteads (that offers lighting to Maasai bomas as well as scaring away potential carnivores at night) and expansion of tolerance for carnivores through consolation payments for livestock killed (but weighed against fence maintenance and structural integrity) needs more research to add to that of Maclennan et al. (2009 [36]). This will provide answers and more insights into carnivore responses to these mitigation approaches similar to this study. It is also critical that we continue monitoring predator numbers and their distribution (Ogutu 1994 [37]), which will also help determine the frequency of encounter with Maasai homesteads and livestock so as to help predict future predation conflicts. Homesteads where predator proof fences are 
needed should be chosen on the basis of severity and prevalence of livestock attacks, particularly the number of cattle and shoats killed per month (i.e. economic loss to two most socially and economically important livestock types), the hyena and lion kills (most common carnivore problem animals) and the number of fence repairs undertaken per month (vulnerability). Based on this study, we recommend that the Maaasai take more responsibility in boma fence maintenance, and ensure that this is done well, more frequently and uses high quality material so that the effectiveness of the fence is maintained. They should also be encouraged to find alternative materials to fence their bomas by investing in boma proof fence that uses chain link wire rather than using thorny woody plant branches which deplete such resources and in the long term increase habitat degradation. To further prevent livestock depredation by carnivores, it's advisable that Maasai adult males and warriors be more vigilant at night as an additional deterrence of predation, but restrain themselves from retaliatory killing of carnivores. They should also be supported to explore initiatives such as predator proof fencing and installation of flickering lights around the bomas as a means of preventing livestock predation, and by so doing help reduce use and dependency on funds for the carnivore consolation scheme administered by Big Life Foundation.

In terms of research, we recommend experimental research that will work if predator proof fencing and light sensor motion individually or in combination can provide better prevention of livestock predation by carnivores. We also encourage exploration of the potential multiplier effect of the Maasai men being involved in more night vigilance and how that would help reduce predation effects on livestock in the bomas at night. It's also important to engage in monitoring and mapping out types of carnivore conflicts and species involved, method and process of attacks, hotspots and spatial-temporal prediction of conflicts. Establishing number, density and spatial distribution of common carnivores (hyenas, lions, jackals, leopards and cheetahs) in core use areas (national parks and sanctuaries) and in dispersal areas should continue. Further, there's a need to monitor their numbers and population dynamics, landscape level movements and the effect of habitat fragmentation and human encroachment on carnivore survival.

\section{Acknowledgements}

We thank the African Wildlife Foundation (AWF) for funding this work and providing transport (vehicles) during the data collection process, and in particular Fiesta Warinwa's encouragement and support throughout this project. The School for Field Studies (and its Center for Wildlife Management Studies in Kenya) is equally appreciated for providing logistical and manpower support. We are also greatly indebted to all the field data collection staff: Ernest Lenkoina, Moses Waema, Daniel Moonka, Francis Nkadayo, and Rana Waikoi; and the AWF drivers: Nichodemus Masila and Stephen Narasha. The leadership of Elerai Conservancy and its members were very instrumental and supportive of this research. In particular, we thank Elerai Conservancy Chairman (Peter Parmeteu), Secretary (Jonah Marahpash) and Treasurer (Parattei Kimiti). We also thank the leadership of Oltiyiani Conservancy and its membership, especially the Chairman (Lekala Ole Kumari), the Vice Chairman (AlaisOle Kisongoi), Secretary (Douglas Meritei) and the Titai Lokida (Treasurer). We equally thank and appreciated the support and input from Mr. Wilfred Gonze who is the Elerai Conservancy warden, and all the community game scouts and other land owners who participated in discussions with the principla investigators in both Elerai and Olitiyiani conservancies. Finally, we thank all those who contributed to intellectual input of this work.

\section{References}

[1] Kenya Wildlife Service (KWS) (1994) Executive Summary: Human Wildlife Conflicts in Kenya. Kenya Wildlife Service, Nairobi.

[2] Woodroffe, R. (2000) Predators and People: Using Human Densities to Interpret Declines of Large Carnivores. Animal Conservation, 3, 165-173. http://dx.doi.org/10.1111/j.1469-1795.2000.tb00241.x

[3] Patterson, B.D., Kasiki, S.M., Selempo, E. and Kays, R.W. (2004) Livestock Predation by Lions (Panthera leo) and other Carnivores on Ranches Neighboring Tsavo National Parks, Kenya. Biological Conservation, 119, 507-516. http://dx.doi.org/10.1016/j.biocon.2004.01.013

[4] Maclennan, S., Groom, R., Macdonald, D.W. and Frank, L. (2009) Evaluation of Compensation Scheme to Bring about Pastoralist Tolerance of Lions. Biological Conservation, 14, 2419-2427. http://dx.doi.org/10.1016/j.biocon.2008.12.003

[5] Norton-Griffiths, M. and Southey, C. (1995) The Opportunity Costs of Biodiversity Conservation in Kenya. Ecological 
Economics, 12, 125-139. http://dx.doi.org/10.1016/0921-8009(94)00041-S

[6] Newmark, W.D. (1994) The Conflicts Between Wildlife and Local People Living Adjacent to Protected Areas in Tanzania: Human Density as a Predictor. Conservation Biology, 8, 249-255. http://dx.doi.org/10.1046/j.1523-1739.1994.08010249.x

[7] Frank, L.G. (2004) Living with Lions: Carnivore Conservation and Livestock in Laikipia District, Kenya. Biological Conservation, 119, 507-516.

[8] Kimani, K. and Pickard, J. (1998) Recent Trends and Implications of Group Ranch Sub-Division and Fragmentation in Kajiado District, Kenya. The Geographical Journal, 164, 202-218. http://dx.doi.org/10.2307/3060370

[9] Okello, M.M. and Kiringe, J.W. (2004) Threats to Biodiversity and Their Implications in Protected and Adjacent Dispersal Areas of Kenya. Journal of Sustainable Tourism, 12, 55-69. http://dx.doi.org/10.1080/09669580408667224

[10] Hazzah, L., Mulder, M.B. and Frank, L. (2009) Lions and Warriors: Social Factors Underlying Declining African Lion Populations and the Effect of Incentive-Based Management in Kenya. Biological Conservation, 142, 2428-2437. http://dx.doi.org/10.1016/j.biocon.2009.06.006

[11] Gowdy, M.J. (1997) The Value of Biodiversity: Markets, Society, and Ecosystems. Land Economics, 73, 25-41. http://dx.doi.org/10.2307/3147075

[12] Wishitemi, B.E.L. and Okello, M.M. (2003) Application of the Protected Landscape Model in Southern Kenya. Parks, 13, 12-21.

[13] Morell, V. (1993) Surrounded! Civilization Is Encroaching on Nairobi National Park (Nairobi Wild Side). International Wildlife, 27, 38-44.

[14] Newmark, W.D. (1993) The Role and Designing of Wildlife Corridors and Examples From Tanzania. Ambio, 22, 500-504.

[15] Newmark, W.D. (1996) Insularization of Tanzanian Parks and the Local Extinction of Large Mammals. Conservation Biology, 10, 1549-1556. http://dx.doi.org/10.1046/j.1523-1739.1996.10061549.x

[16] Stuart-Hill, G. and Grossman, D. (1993) Parks, Profits and Professionalism: Lion Return to Pilanesberg. African Wildlife, 47, 267.

[17] Graham, O. (1989) A Land Divided: The Impact of Ranching on a Pastoral Society. Ecologist, 19, 184-185.

[18] Hackel, J.D. (1998) Community Conservation and the Future of Africa's Wildlife. Conservation Biology, 13, 726-734. http://dx.doi.org/10.1046/j.1523-1739.1999.98210.x

[19] Ferraro, P.J. and Kiss, A. (2000) Direct Paymentst to Conserve Biodiversity. Science, 298, 1718-1719.

[20] Okello, M.M., Seno, S.S. and Wishtemi, B.B. (2003) Maasai Community Wildlife Sanctuaries in Tsavo-Amboseli, Kenya. Parks, 13, 62-75.

[21] Campbell, D.J., Gichohi, H., Mwangi, A. and Chege, L. (2000) Land Use Conflict in Kajiado District, Kenya. Land Use Policy, 17, 337-348. http://dx.doi.org/10.1016/S0264-8377(00)00038-7

[22] Mizutani, F. (1993) Home Range of Leopards and Their Impact on Livestock on Kenyan Ranches. In: Dunstone, N. and Gorman, M.L., Eds., Mammals as Predator, Clarendon Press, Oxford, 425-439.

[23] Butler, J.R.A. (2000) The Economic Costs of Wildlife Predation on Livestock in Gokwe Communal Land, Zimbabwe. African Journal of Ecology, 38, 23-30. http://dx.doi.org/10.1046/j.1365-2028.2000.00209.x

[24] Kerbis, A., Peterhans, J.C. and Gnoske, T.P. (2002) The Science of Man-Eating among Lions Panthera Leo with Reconstruction of the Natural History of the Man-Eaters of Tsavo. Journal of East African Natural History, 90, 1-40. http://dx.doi.org/10.2982/0012-8317(2001)90[1:TSOMAL]2.0.CO;2

[25] Hazzah, L., Dolrenry, S., Kaplan, D. and Frank, L. (2013) The Influence of Park Access during Drought on the Attitudes towards Wildlife and Lion Killing Behavior in Maasailand, Kenya. Environmental Conservation, 40, 266-276.

[26] Galaty, J.G. (1992 This Land Is Yours: Social and Economic Factors in the Privatization, Subdivision and Sale of Maasai Ranches. Nomadic Peoples, 30, 26-40.

[27] Western, D. (1975) Water Availability and Its Influence on the Structure and Dynamics of a Savannah Large Mammal Community. East African Wildlife Journal, 13, 265-286. http://dx.doi.org/10.1111/j.1365-2028.1975.tb00139.x

[28] Rudnai, J. (1979) Ecology of Lions in Nairobi National Park and the Adjoining Kitengela Conservation Unit in Kenya. African Journal of Ecology, 17, 85-95. http://dx.doi.org/10.1111/j.1365-2028.1979.tb00460.x

[29] Ntiati, P. (2002) Group Ranches Subdivision Study in Loitokitok Division of Kajiado District, Kenya. Lucid Working Paper Series, 7, 1-26.

[30] Irigia, B.K. (1995) Kenya Wildlife Service Environmental Impact Assessment of the Proposed Kimana Wildlife Sanctuary. Community Wildlife Service, KWS, Nairobi, 29.

[31] Gachimbi, L.N. (2002) Technical Report of Soil Survey and Sampling: Loitokitok Division, Kajiado District. LUCID 
Working Paper Series, 10, 1-22.

[32] Okello, M.M. (2009) Contraction of Wildlife Dispersal Area and Displacement by Human Activities in Kimana Group Ranch near Amboseli N. Park. The Open Conservation Biology Journal, 3, 49-56. http://dx.doi.org/10.2174/1874839200903010049

[33] Zar, J.H. (1999) Biostatistical Analysis. 4th Editon, Prentice-Hall, Upper Saddle River.

[34] Estes, R.D. (1997) The Behavior Guide to African Mammals. Russel Friedman Books.

[35] Kiringe, J.W. and Okello, M.M. (2005) Use and Availability of Tree and Shrub Resources on Maasai Communal Rangelands near Amboseli, Kenya. African Journal of Range and Forage Science, 22, 37-46. http://dx.doi.org/10.2989/10220110509485860

[36] Maclennan, S.D., Groom, R.J., Macdonald, D.W. and Frank, L.G. (2009) Evaluation of Compensation Scheme to Bring about Pastoralist Tolerance of Lions. Biological Conservation.

[37] Ogutu, J.O. (1994) Test of a Call-In Technique for Estimating Lion (Panthera leo, Linnaeus 1758) Population Size in the Masai Mara National Reserve, Kenya. M.Phil. Thesis, Department of Wildlife Management, Moi University, Eldoret. 
Scientific Research Publishing (SCIRP) is one of the largest Open Access journal publishers. It is currently publishing more than 200 open access, online, peer-reviewed journals covering a wide range of academic disciplines. SCIRP serves the worldwide academic communities and contributes to the progress and application of science with its publication.

Other selected journals from SCIRP are listed as below. Submit your manuscript to us via either submit@scirp.org or Online Submission Portal.
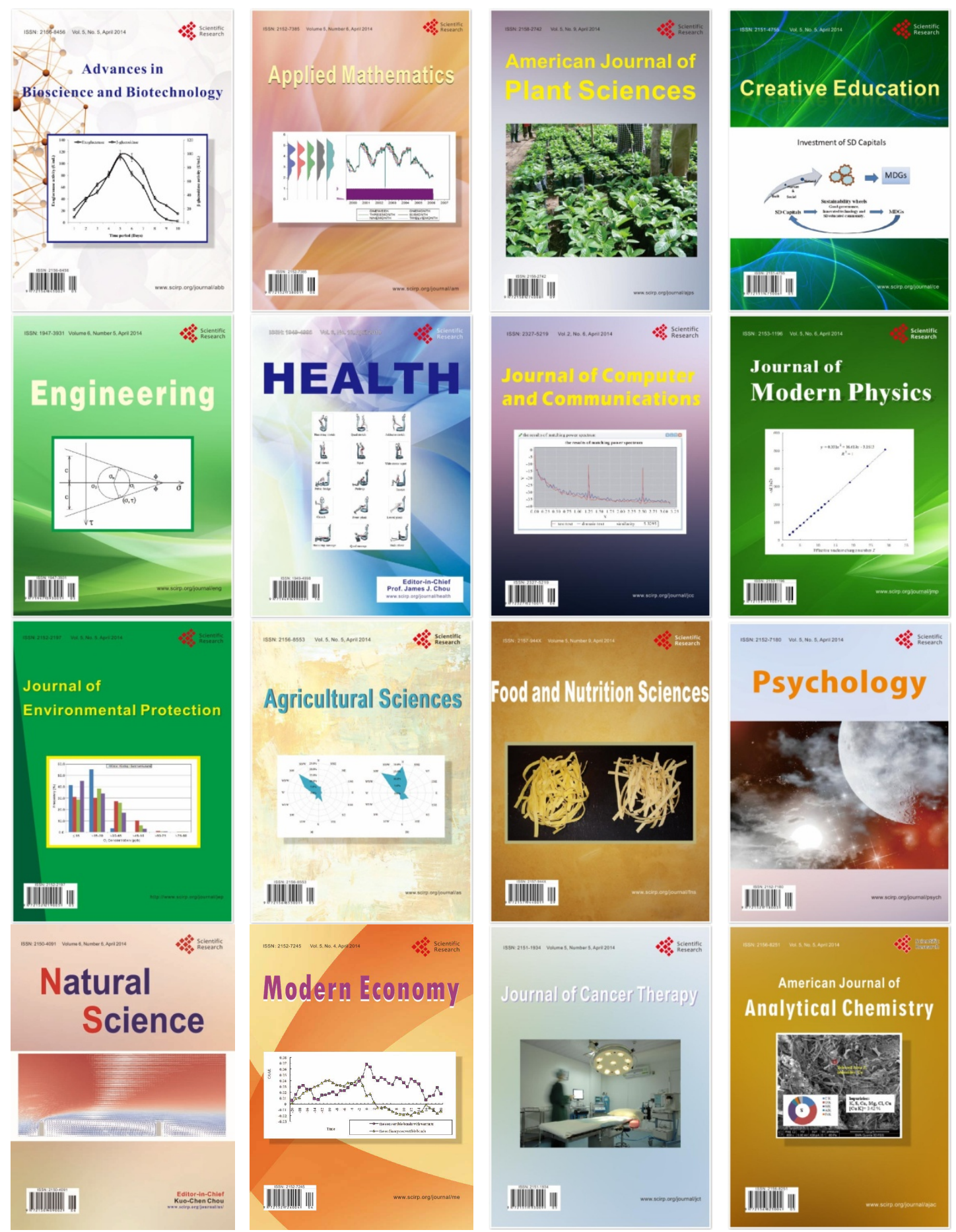